\title{
Sulla approssimabilità dei numeri algebrici mediante numeri razionali.
}

\author{
Memoria di Manco Cugrani (a Milano)
}

A Giovanni Sansone nel suo 70mo compleanno.

Sunto. - Sfruttando il metodo di RoTH-SchneIder si dimostra il seguente teorema: « Se $\alpha$ $\dot{e}$ algebrico di grado $g>1$ e se esiste una successione $\left|p_{n}\right| q_{n} \mid$ di numeri razionali $\left(0<q_{n} \leq q_{n+1}\right)$, per cur si ha: $q_{n}=q_{n}^{\prime} \cdot q_{n}{ }^{\prime \prime}$, essendo $q_{n}{ }^{\prime \prime}=b^{\lambda n}$, in modo che risulti $\log q_{n}^{\prime \prime} / \log q_{n} \geq 1-\eta-\varphi\left(q_{n}\right) \cdot \omega(\eta$ ed $\omega$ costanti $\geq 0)$ ed inoltre:

$$
\begin{aligned}
& \left|\alpha-p_{n} / q_{n}\right|<q_{n}-\left(1+n+f\left(q_{n}\right)\right. \\
& \text { dove } \varphi\left(q_{n}\right)=\left(\log \log \log q_{n}\right)^{-\frac{1}{2}}, f\left(q_{n}\right)=\left\{(1+2 \eta)(2 g+1)+\omega+\varepsilon \mid \varphi\left(q_{n}\right)\right. \\
& \text { con } \varepsilon>0, \text { allora sarà: }
\end{aligned}
$$

$$
\varlimsup_{n \rightarrow+\infty} \log q_{n+1} / \log q_{n}=+\infty
$$

\section{I. - Introduzione - Risultati.}

Del risultato ormai classico di K. F. ROTH $\left({ }^{1}\right)$, sulla approssimabilità di un numero algebrico mediante razionali, sono state date parecchie varianti. Ricordiamo qui in particolare quella dovuta a TH. Schneider $\left({ }^{2}\right)$ la quale conduce a una proposizione del seguente tenore:

«se $\alpha$ è algebrico di grado $g>1$ e $\left\{p_{n} / q_{n}\right\}$ ̀े una successione di numeri razionali, con $\left(p_{n}, q_{n}\right)=1, q_{n+1} \geq q_{n}>0$, i cui denominatori si possano scom. porre nella forma: $q_{n}=q_{n}^{\prime} \cdot q_{n}^{\prime \prime}$, dove $q_{n}^{\prime \prime}=b^{\lambda_{n}}$ con $b$ intero fisso $e \lambda_{n}$ intero; allora, posto

$$
\eta=\varlimsup_{n \rightarrow \infty} \frac{\log q_{n}^{\prime}}{\log q_{n}}
$$

e scelto $\mu>1+\eta$ e del resto qualunque, esiste al più un numero finito di frazioni $p_{n} / q_{n}$ per le quali risulta:

$$
\left|\alpha-p_{n} / q_{n}\right|<q_{n}^{-\mu} . »
$$

(1) Si veda: K. F. Rotr, Rationa' approximation to algebraic numbers, a Mathematika , $2(1955), 1.20$.

(i) Si veda: Th, SchNeIDer, Einfürung in die transzendenten Zahlen, "Grund. Math. Wiss. 81 , Springer, Berlin (1957). Qui si allude al Satz 6, § 5, pag. 13. 
Poichè è evidentemente $\eta \leq 1$ in ognni caso, dalla proposizione sopra enunciata viene implicato anche il teorema di Rotн.

Noi vogliamo dare del teorema di Rom-Schneider una nnova variante, giungendo a dimostrare il seguente:

Teonema. - Sia a algebrico di grado $g>1$, sia poi assegnata una successione $\left\{p_{n} \mid q_{n}\right\}$, con $q_{n+1} \geq q_{n}>0,\left(p_{n}, q_{n}\right)=1$, tale che $i q_{n}$ si possano decomporre nel modo seguente:

$$
q_{n}=q_{n}^{\prime} \cdot q_{n}^{\prime \prime}, q_{n}^{\prime \prime}=b^{\lambda_{n}}
$$

con $b$ e $\lambda_{n}$ interi, $b$ fisso, la quale inoltre soddisfaccia le due condizioni:

a) esistano due costanti $\eta$ ed $\omega(0 \leq \eta \leq 1,0 \leq \omega)$ tali che risulti

$$
\frac{\log q^{\prime \prime} n}{\log q_{n}} \geq 1-\eta-\left(\log \log \log q_{n}\right)^{1 / 2}
$$

b) esista un $\varepsilon>0$, abbastanza piccolo, per cui si abbia:

$$
\left|\alpha-\frac{p_{n}}{q_{n}}\right|<q_{n}-\left(1+n+f\left(q_{n}\right)\right)
$$

essendo:

$$
f\left(q_{n}\right)=\{(4+2 \eta)(2 g+1)+\omega+\varepsilon\} /\left(\log \log \log q_{n}\right)^{\frac{1}{2}}
$$

risulterò allora:

$$
\varlimsup_{n \rightarrow \infty} \frac{\log q_{n+1}}{\log q_{n}}=+\infty
$$

Da questo teorema si deduce un criterio di trascendenza. in base al quale si può affermare ad esempio che sono trascendenti tutti i numeri $\alpha$ della famiglia :

$$
\alpha=\sum_{\nu=0}^{\infty} d, \alpha-c^{\nu}
$$

con $a, c$ interi fissi, $a \geq 2, c \geq 2$, essendo $i d$, interi, divorsi da zero per infiniti valori di $v$, chiamiamoli $\psi^{*}$, abbastanza fitti perchè la differenzn di due successivi $\vee^{*}$ risulti sempre minore di una costante fissa; per $\vee$ abbastanza grande sia inoltre:

$$
\left|d_{\nu}\right| \leq a^{c^{\nu-1}}\left\{1-[\log (\nu-1)]^{-\frac{1}{2}+\varepsilon}\right\}
$$

per un $\varepsilon>0$ fisso.

Arrestandoci infatti al termine n-esimo (is corrispondenza di un $d_{n} \neq 0$ ), e ponendo:

$$
p_{n} / q_{n}=\sum_{y=0}^{n} d_{2} a-c^{2}
$$


avremo, essendo ovviamente ogni termine non nullo maggiore del doppio di ognuno dei successivi (per $\vee$ abbastanza grande):

$$
\left|\alpha-p_{n} / q_{n}\right|=\sum_{\nu=n+1}^{\infty} d_{y} a-c^{\nu}<2 d_{n+1} a-e^{n+1}
$$

(nell' ultimo membro si può serivere $n+r$ invece di $n+1$ se risulta $d_{n+1}=d_{n+2}=\ldots=d_{n+r-1}=0, d_{n+r} \neq 0$, il che non altera sostanzialmente il ragionamento che segue). Abbiamo ora:

$$
2 d_{n+1} a-c^{n+1} \leq a^{-\left(c^{n+1}-c^{n}+\frac{c^{n}}{(\log n)^{1 / 2}-\varepsilon}-\log _{a} 2\right)}
$$

ricordando che: $q_{n} \leq a c^{n}$, e ponendo:

otteniamo

$$
q_{n}^{X}=a^{e^{n+1}-c^{n}+-\frac{e^{n}}{(\log n)^{1 / 2-\varepsilon}}-\log _{a} 2}
$$

ed essendo $c \geq 2$, ne viene

$$
X \geq c-1+\frac{1}{(\log n)^{1 / 2-\varepsilon}}+O\left(\frac{1}{c^{n}}\right)
$$

$$
X \geq 1+\frac{1+o(1)}{(\log n)^{1 / 2-\varepsilon}}
$$

Qui possiamo prendere $\eta=0$ ed $\omega=0$ e la (1) sarà sempre soddisfatta essendo $q_{n}{ }^{\prime \prime}=q_{n}$, mentre troviamo subito:

$$
\begin{gathered}
\varlimsup_{n \rightarrow \infty} \frac{\log q_{n+1}}{\log q_{n}} \leq \\
\leq \lim \frac{c^{n+1} \log a}{c^{n-1} \log a}=c^{2}<+\infty .
\end{gathered}
$$

Ora se $\alpha$ fosse algebrico di grado $g$, avendosi:

$$
\log \log \log q_{n}=\log n+O(1) ;\left(\log \log \log q_{n}\right)^{\frac{1}{2}}=(\log n)^{\frac{1}{2}}+O(1)
$$

non potrebbe essere, a causa della (2), per un $K$ opportuno:

$$
\mathrm{X}<1+\frac{K}{(\log n)^{1 / 2}+o(1)}
$$

in evidente contrasto colla (4) per $n$ abbastanza grande.

Ovviamente il precedente discorso si può applicare con lievi modificazioni più in generale a numeri del tipo:

$$
\alpha=\Sigma \frac{d_{\nu}}{a c^{\nu}} x^{\nu}
$$

per ogni $x$ razionale e colle stesse limitazioni per $a, c, d_{\nu}$; I numeri $\alpha$ del tipo $\left(3^{\prime}\right)$ risultano dunque anch' essi trascendenti. 


\section{II. - Due lemmi preliminari.}

Per la dimostrazione del nostro teorema ci serviremo di due lemmi che desumiamo dai lavori di RotH, e di SchNerder, e al cui enunciato dovremo premettere un breve richiamo della nozione di indice di un polinomio in un punto, la quale del resto è ormai classica in questo tipo di ricerche.

Sia $Q\left(x_{1}, x_{2}, \ldots, x_{m}\right)$ un polinomio non identicamente nullo, ed inoltre siano $\alpha_{1}, \alpha_{2}, \ldots, \alpha_{m}$ ed $r_{1}, r_{2}, \ldots, r_{m}$ due $m$-ple di numeri reali $\left(r_{1}>0\right)$. Poniamo:

$$
Q\left(\alpha_{1}+y_{1}, \ldots, \alpha_{m}+y_{m}\right)=\Sigma c_{i_{1}, i_{2}, \ldots, i_{m}} y_{1}^{i_{1}} y_{2}^{i_{2}} \ldots y_{m}^{i_{m}}
$$

essendo le $y_{i}$ nuove variabili. Diremo allora indice del polinomio $P$ nel punto $A \equiv\left(\alpha_{1}, \alpha_{2}, \ldots, \alpha_{m}\right)$ in relazione alla $m$-pla $r_{1}, r_{2}, \ldots r_{m}$, il numero $\theta$, cosi definito:

$$
\theta=\underset{\left(\begin{array}{l}
i_{1}, i_{2}, \ldots \ldots, i_{m} \\
i_{i_{1}}, i_{2}, \ldots, i_{m} \neq 0
\end{array}\right)}{\operatorname{Min}}\left(\frac{i_{1}}{r_{1}}+\frac{i_{2}}{r_{2}}+\ldots+\frac{i_{m}}{r_{m}}\right) .
$$

Indichiamo poi con:

$$
\theta_{m}\left(B ; q_{1}, q_{2}, \ldots, q_{m} ; r_{1}, r_{2}, \ldots, r_{m}\right)
$$

(dove gli argomenti sono suscettibili di valori reali positivi e di più $i q_{i}$ interi) l'estremo superiore degli indici $\theta$ nei punti $A\left(\frac{p_{2}}{q_{2}}, \frac{p_{2}}{q_{2}}, \ldots, \frac{p_{m}}{q_{m}}\right)$, mentre variano:

(a) la $m$-pla $p_{1}, p_{2}, \ldots, p_{m}$, nella quale ogni numero $p_{i}$ assumerà tutti i valori interi compatibili colla condizione $\left(p_{i}, q_{i}\right)=1$;

b) il polinomio $Q$, non identicamente nullo, i cui coefficienti, interi, assumeranno tutti $i$ valori compatibili colla condizione che il grado di $Q$ ri. spetto ad ogni $x_{i}$ non superi $r_{i}$, mentre il valore assoluto dei coefficienti stessi non supera $B$.

Passiamo ora ad enunciare il: $\left({ }^{3}\right)$

Lemma $1^{\circ}$. - Sia $m$ intero positivo e $\delta$ reale, tali che:

$$
0<\delta<1 / m
$$

$r_{1}, r_{2}, \ldots, r_{m}$ siano numeri interi positivi per $i$ quali si ha:

$$
\begin{gathered}
r_{m}>10 / \delta \\
r_{j-1} / r_{j}>1 / \delta \quad j=2,3, \ldots, m .
\end{gathered}
$$

() Si veda: K. F. RoTh, loc. eit. in (1), Lemma 7, alla pag. 12. 
I numeri $q_{1}, q_{2}, \ldots, q_{m}$ interi soddisfino alle relazioni:

$$
0<q_{1} \leq q_{2} \leq \ldots \leq q_{m}
$$

$$
\log q_{1}>m(2 m+1) / \delta
$$

$$
r_{j} \log q_{j} \geq r_{1} \log q_{1} \quad(j=2,3, \ldots, m) \text {; }
$$

allora si ha:

$$
\theta_{m}\left(q_{1}^{\delta r_{2}} ; q_{1}, q_{2} \ldots, q_{m} ; r_{1}, r_{2}, \ldots, r_{m}\right)<10^{m \delta}{ }^{\left(\frac{1}{2}\right)^{m}}
$$

E passiamo adesso a esporre il ( $\left.{ }^{4}\right)$ :

LEMma $2^{\circ}$. - Sia $\propto$ algebrico di grado g. Fissati un numero reale $\tau$, e un numero $m$ intero, tali che risulti:

$$
0<\tau<\frac{1}{2} \quad \tau m^{\frac{1}{2}}-1>2 g
$$

allora, comunque si scelga una m-pla di numeri interi positivi $r_{1}, r_{2}, \ldots, r_{m}$, si può sempre trovare un polinomio, non identicamente nullo, diciamo $\Phi\left(x_{1}, x_{2}, \ldots, x_{m}\right)$ a coefficienti razionali interi, colle seguenti proprietà :

a) tutte le derivate:

$$
\left(\frac{\partial}{\partial x_{1}}\right)^{i_{1}}\left(\frac{\partial}{\partial x_{2}}\right)^{i_{2}} \cdots\left(\frac{\partial}{\partial x_{m}}\right)^{i_{m}} \Phi
$$

$\operatorname{con}$

$$
\frac{i_{1}}{r_{1}}+\frac{i_{2}}{r_{2}}+\ldots+\frac{i_{m}}{r_{m}} \geq m\left(\frac{1}{2}+\tau\right)
$$

sono identicamente nulle;

b) l'indice $d i \Phi$ nel punto $A(\alpha, \alpha, \ldots, \alpha)$ in relazione alla m-pla $r_{1}$, $r_{2}, \ldots, r_{m} \grave{e}$ maggiore di:

$$
m\left(\frac{1}{2}-\tau\right)
$$

c) esiste una costante $\gamma$ dipendente solo da $\alpha$ per cui si ha:

$$
e^{\gamma\left(r_{1}+r_{2} \ldots+r_{m}\right)} \geq B
$$

essendo $B$ il massimo modulo dei coefficienti $d i \Phi$.

(4) Si veda: Th. Sohneider, op. cit. in ( $\left.{ }^{2}\right)$, § 5, Hilfssatz 11, pag. 26. 


\section{III - Determinazione dei parametri.}

Prima di procedere alla dimostrazione vera e propria del nostro teorema, dovremo far vedere come i parametri $\tau, m, \delta, r_{1}, r_{2}, \ldots, r_{m}, q_{1}, \ldots, q_{m}$ possono essere scelti in guisa che risultino soddisfatte tutte le condizioni poste nei precedenti lemmi, nonchè le altre che verranno qui di seguito indicate e che risulteranno essenziali ai fini della dimostrazione.

Procederemo per assurdo e quindi cominceremo col supporre che contrariamente alla tesi da dimostrare si possa trovare una costante $\Delta$ per cui risulti :

$$
\frac{\log q_{n+1}}{\log q_{n}}<\Delta
$$

per $n$ abbastanza grande, anzi sarà equivalente il supporre, con una eventuale diversa scelta della costante $\Delta$, che tale relazione sia soddisfatta per ogni $n$. Potremo dunque scrivere:

$$
q_{n+1}<q_{n}^{4}
$$

Poniamo adesso:

$$
\delta=e^{-e^{m}}
$$

e decidiamo di chiamare $q_{1}$ il primo dei numeri $q_{i}$ per i quali si ha:

$$
\log q_{1} \geq e^{2 e^{m}}
$$

ponendo esattamente:

$$
\log q_{1}=c e^{2 e^{m}}
$$

risulterà a causa della (12): $1 \leq c<\Delta$, onde potremo scrivere:

$$
\log \log \log q_{1}=m+O(1) ;\left(\log \log \log q_{1}\right)^{\frac{1}{2}}=m^{\frac{1}{9}}+o(1)
$$

Con tali posizioni saranno inoltre soddisfatte la (5) e la (8) non appena $m$ è abbastanza grande, e in più potremo fare in modo che risulti :

$$
\log q_{1}>m \gamma_{1} / \delta
$$

dove:

$$
\gamma_{1}=\operatorname{Max}(\gamma, \log (16(1+|\alpha|)\})
$$

essendo $\gamma$ la costante che figura nel punto $c$ ) del lemma $2^{\circ}$. 
Scegliamo poi una catena di valori $q_{1}$, e li indicheremo con $q_{j}$, in modo che risulti:

$$
\frac{\log q_{j}}{\log q_{j \sim 1}}>\frac{2}{\delta}=2 e^{e m} \quad(j=2,3, \ldots, m)
$$

e scegliamo ciascun $q_{j}$ in modo che sia il minimo dei $q_{i}$ che soddisfa alla corrispondente relazione (15).

Ne risulterà allora, per la (12):

$$
\log q_{1} / \log q_{j-1} \leq 2 \Delta e^{e m}
$$

e si avrà :

$$
q_{m}<q_{1}(24)^{m \cdot e^{m e m}}
$$

ossia :

$$
\left(\log \log \log q_{m}\right)^{\frac{1}{2}} \leq\left(m+\log m+O\left(\frac{1}{m}\right)\right)^{\frac{1}{2}}=m^{\frac{1}{2}}+o(1) .
$$

Al valore di $m$ prescelto verrà dunque ad essere associata una $m$-pla di denominatori $q_{j}(j=1,2, \ldots, m)$, in modo che oltre la $(5)$ e la (8) sia soddisfatta la (15); se facciamo ulteriormente aumentare $m$, $\theta$ variamo in conse. guenza la $m$-pla dei $q_{j}$, queste tre relazioni saranno sempre soddisfatte; la (13), e la (16), valide per $m \rightarrow+\infty$, ci permettono poi, aumentando eventualmente ancora $m$, di fare in modo che, fissato $\sigma>0$ e posto:

$$
\tau=\frac{2 g+1+\sigma}{\left(\log \log \log q_{m}\right)^{\frac{1}{2}}}
$$

risultino soddisfatte anche le relazioni (11), oltre alle seguenti :

$$
\begin{gathered}
\delta<9 \sigma \tau<\frac{m \sigma \tau}{4} \\
\sigma \tau m>10^{m} \cdot \delta^{(1 / 2)^{m}}
\end{gathered}
$$

$$
\tau\left(\log \log \log q_{j}\right)^{\frac{1}{2}}>2 g+1 \quad(j=1,2, \ldots, m)
$$

$$
+2(1+\eta)(1+\sigma) \tau+O\left(\tau^{2}\right) \leq 1+\eta+\frac{(4+2 \eta)(2 g+1)+\omega+\varepsilon}{\left(\log \log \log q_{m}\right)^{\frac{1}{2}}}=1+\eta+f\left(q_{m}\right)
$$


dove per l'arbitrarietà di $\sigma$, anche $\varepsilon>0$ può essere scelto piccolo a piacere.

Fissato cosi definitivamente $m$, e quindi $\delta$, nonchè la $m$-pla dei $q_{j}$, scegliamo un numero naturale $r_{1}$ tale ohe si abbia:

$$
r_{1}>10 \log q_{m}\left(\delta \log q_{1}\right)
$$

e successivamente altri $m-1$ numeri naturali $r_{j}(j=2,3, \ldots, m)$, definiti dalle relazioni:

$$
r_{1} \log q_{1} / \log q_{1} \leq r_{j}<1+r_{1} \log q_{1} / \log q_{i} .
$$

Grazie alla (22) anche la (9) sarà soddisfatta e, tenendo sonto della (21), sarà soddisfatta anche la (6). Avremo inoltre:

$$
\frac{r_{j} \log q_{s}}{r_{1} \log q_{1}}<1+\frac{\log q_{1}}{r_{1} \log q_{1}} \leq 1+\frac{\log q_{m s}}{r_{1} \log q_{1}}<1+\frac{\delta}{10}
$$

da cui, per la (15), otteniamo:

$$
\frac{r_{j-1}}{r_{j}}>\frac{\log q_{j}}{\log q_{j-1}}\left(1+\frac{\delta}{10}\right)^{-1}>\frac{1}{\delta}
$$

onde anche la (7) sarà soddisfatta.

\section{IV. - Dimostrazione del teorema.}

Fissati cosi $i$ valori dei parametri $m, \delta, \tau, q_{1}, q_{2}, \ldots, q_{m}, r_{1}, r_{2}, \ldots, r_{m}$ in modo che siano soddisfatte tutte le condizioni per la validità dei Lemmi $1^{\circ}$ e $2^{\circ}$, costruiamo un polinomio $\Phi$ colle proprietà indicate nel lemma $2^{\circ}$.

Il polinomio $\Phi\left(x_{1}, x_{2}, \ldots, x_{m}\right)$ potrebbe non servire al nostro scopo in quanto potrebbe annullarsi nel punto $\left(\frac{p_{1}}{q_{1}}, \frac{p_{2}}{q_{2}}, \ldots, \frac{p_{m}}{q_{m}}\right)$.

Osserviamo allora che per il lemma $2^{\circ}$, comma $c$ ), e per la (7), avremo:

$$
B \leq e r^{\left(r_{1}+r_{3}+\ldots+r_{m}\right)}<e^{m r_{1}}
$$

e quindi per la (14):

$$
B<q_{1}^{8 r_{1}}
$$

Allora, per la (10) del lemma $1^{\circ}$, e per la (18) avremo:

$$
\theta_{m}<10^{m \delta}\left(\frac{1}{2}\right)^{m}<\sigma \tau m
$$

Esiste quindi almeno una $m$-pla di numeri interi non negativi $i_{1}, i_{2}, \ldots, i_{m}$, per cui si ha:

$$
\frac{i_{1}}{r_{1}}+\frac{i_{2}}{r_{2}}+\ldots+\frac{i^{m}}{r_{m}}<\sigma \tau m
$$


e tale che il polinomio corrispondente:

$$
F\left(x_{1}, x_{2}, \ldots, x_{m}\right)=\frac{1}{i_{1} ! i_{2} ! \ldots i_{m} !}\left(\frac{\partial}{\partial x_{1}}\right)^{i_{1}}\left(\frac{\partial}{\partial x_{2}}\right)^{i_{2}} \ldots\left(\frac{\partial}{\partial x_{m}}\right)^{i_{m}} \Phi
$$

non si annulla nel punto $A\left(p_{1} / q_{1}, p_{2} / q_{2}, \ldots, p_{m} / q_{m}\right)$.

Il polinomio $F\left(x_{1}, x_{2}, \ldots, x_{m}\right)$ arrà coefficienti razionali interi, per il modo stesso con cui è costruito, inoltre detto $B_{1}$ il massimo modulo di tali coefficienti, ricordando la limitazione $\left(\begin{array}{l}n \\ k\end{array}\right) \leq 2^{n}$, si vede subito che sarà $B_{1}<$

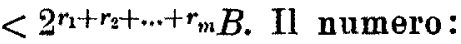

$$
F=\left|F\left(\frac{p_{1}}{q_{1}}, \frac{p_{2}}{q_{2}}, \ldots, \frac{p_{m}}{q_{m}}\right)\right|>0
$$

sarà dunque razionale, ed arremo inoltre che, scritto in forma ridotta, arrà per denominatore un divisore del numero:

$$
q_{1}^{r_{1}} q_{2}^{r_{3}} \ldots q_{m 3}^{r_{m}}
$$

Ora abbiamo per (1) e (19):

$$
\frac{\lambda_{j} \log b}{\log q_{j}} \geq 1-\eta-\frac{\omega}{\left(\log \log \log q_{j}\right)^{\frac{1}{z}}}>1-\eta-\frac{\omega \tau}{2 g+1}=1-\eta-\Omega .
$$

In $q_{1}^{r_{1}} \cdot q_{2}^{r_{3}} \ldots q_{m}^{r m} \dot{\theta}$ contenuto come fattore il numero

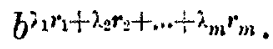

D'altra parte, a causa del lemma 20 , comma $a$ ), ogni termine del polinomio $F\left(x_{1}, x_{2}, \ldots, x_{m}\right)$ dopo le sostituzioni $x_{j} \rightarrow p_{j}^{\prime} q,(j=1,2, \ldots . m)$ si trasforma in una frazione il cui denominatore non puo contenere un prodotto di potenze:

per cui risulti

$$
q_{1}^{i_{1}} \cdot q_{2}^{i_{2}} \cdots q_{m}^{i_{m}}
$$

$$
\frac{i_{1}}{r_{1}}+\frac{i_{2}}{r_{2}}+\ldots+\frac{i_{m}}{r_{m}} \geq m\left(\frac{1}{2}+\tau\right)
$$

Quindi se seriviamo il denominatore del numero $F$ nella forma:

$$
\Pi q_{j}^{\prime s_{j}} \Pi \Pi q_{j}^{\prime s_{j}}=Q^{\prime} \cdot Q^{\prime \prime} \quad\left(s_{j} \leq r_{j}\right)
$$

il fattore $Q^{\prime \prime}$ non puo superare $b l$, dove:

$$
\Lambda=\underset{\left(\Sigma \frac{i_{j}}{r_{j}}<m\left(\frac{1}{2}+\tau\right)\right)}{\operatorname{Max}} i_{1} \lambda_{1}+i_{2} \lambda_{2}+\ldots+i_{m} \lambda_{m} .
$$


Il denominatore di $F$ non può dunque superare la quantità

$$
q_{1}^{r_{1}} \cdot q_{2}^{r_{2}} \ldots q_{m}^{r_{m} / b^{\lambda_{1}} r_{1}+\lambda_{2} r_{2}+\ldots+\lambda_{m} r_{m}-\Lambda} \text {. }
$$

Ora, posto:

$$
H=b^{\sum_{1}^{m}\left(r_{j}-i_{j}\right) \lambda_{j}}
$$

al variare di $i_{1}, i_{2}, \ldots, i_{m}$, sempre sotto la condizione:

$$
\sum_{j=1}^{m} \frac{i_{j}}{r_{j}}<m\left(\frac{1}{2}+\tau\right)
$$

avremo per la (25) e la (9):

$$
H \geq\left\{\prod_{j=1}^{m} q_{j}^{r_{j}-i_{j}}\right\}^{1-\eta-Q} \geq q_{1}\left(\Sigma \frac{r_{j}-i_{j}}{r_{j}}\right)(1-\eta-Q) r_{i} \geq q_{1}{ }^{m}\left(\frac{1}{2}-\tau\right)(1-\eta-Q) r_{i}
$$

e quindi

$$
b^{\Sigma \lambda_{j} r_{j}-\Delta}=\operatorname{Min} H \geq q_{1}^{m}\left(\frac{1}{2}-\tau\right)(1-\eta-Q) r_{1} .
$$

Il denominatore di $F$ sarà dunque non maggiore di

$$
q_{1}^{r_{1}} q_{2}^{r_{z}} \ldots q_{m}^{r_{n}} / q_{2}^{m}\left(\frac{1}{2}-\tau\right)(1-\eta-\Omega) r_{1}
$$

Ora a causa di (22) e di (6) possiamo scrivere:

$$
\frac{r_{j} \log q_{j}}{r_{1} \log q_{1}}<1+\frac{1}{r_{j}-1} \leq 1+\frac{1}{r_{m}-1}<1+\frac{\delta}{9}
$$

e per la (17) avremo anche:

e quindi:

$$
r_{j} \log q_{j}<(1+\sigma \tau) r_{1} \log q_{1}
$$

$$
q_{1}^{r_{1}} \cdot q_{2}^{r_{2}} \ldots q_{m}^{r_{m}}<q_{1}^{m(1+\sigma \tau) r_{1}} .
$$

Potremo allora scrivere (essendo ovviamente il numeratore di $F$ almeno uguale ad 1):

$$
F>q_{1}^{m\left(\frac{1}{2}-\tau\right)(1-\eta-Q) r_{1}-m(1+\sigma \tau) r_{1}}>q_{1}-\frac{m}{2}(1+\eta+2(1+\sigma) \tau+Q) r_{1}
$$

Vogliamo adesso trovare un valore approssimato dal di sopra per il numero $F$.

Pensiamo allo sviluppo di $F\left(x_{1}, x_{2}, \ldots, x_{m}\right)$ per le potenze di $\left(x_{1}-\alpha\right)$, $\left(x_{2}-\alpha\right), \ldots,\left(x_{m}-\alpha\right)$. 
A causa del lemma $2^{\circ}$, comma $b$ ) e della (24) ogni termine di tale sviluppo contiene un fattore

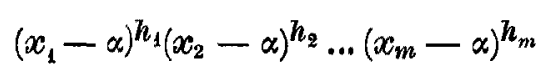

per il quale è certamente:

$$
\frac{h_{1}}{r_{1}}+\frac{h_{2}}{r_{2}}+\ldots+\frac{h_{m}}{r_{m}}>m\left(\frac{1}{2}-(1+\sigma) \tau\right)
$$

Ponendo in tale fattore $x_{j}=p_{j} / q_{j}(j=1,2, \ldots, m)$ avremo intanto a causa di (2) e (9):

$$
\begin{aligned}
& \left|\left(\frac{p_{1}}{q_{2}}-\alpha\right)^{h_{1}}\left(\frac{p_{2}}{q_{2}}-\alpha\right)^{h_{2}} \cdots\left(\frac{p_{m}}{q_{m}}-\alpha\right)^{h_{m}}\right|<\prod_{j=1}^{m} q_{j}^{-h_{j}\left\{1+\eta+f\left(q_{j}\right)\right\}} \leq \\
& \leq q_{1}^{-r_{1} z\left(h_{j} / r_{j}\right) \cdot\left\{1+\eta+f\left(q_{m}\right)\right\}}<q_{1}^{-m}\left\{\frac{1}{2}-(1+\sigma) \tau\right\}\left\{1+\eta+f\left(q_{m}\right) \mid r_{1} .\right.
\end{aligned}
$$

Se indichiamo con $K$ it massimo modulo dei coefficienti dello sviluppo di $F\left(x_{1}, x_{2}, \ldots, x_{m}\right)$ per le potenze degli $\left(x_{i}-\alpha\right)$ avremo:

$$
F<K\left(r_{1}+1\right)\left(r_{2}+1\right) \ldots\left(r_{m}+1\right) q_{1}-\frac{m}{2}\left\{1-2(1+\sigma) \tau \mid\left\{1+\eta+f\left(q_{m}\right)\right\} r_{1} .\right.
$$

Per ottenere una maggiorazione di $K$, ricordiamo ancora la nota relazione $\left(\begin{array}{l}n \\ k\end{array}\right) \leq 2^{n}$, onde, detto ancora $B_{1}$ il massimo modulo dei coefficienti di $F$, e posto $a=1+|\alpha|$, avremo:

$$
\begin{gathered}
K<(2 a)^{r_{1}+r_{2}+\ldots+r_{m}}\left(r_{1}+1\right)\left(r_{2}+1\right) \ldots\left(r_{m}+1\right) B_{1}< \\
<(4 a)^{r_{1}+r_{2}+\ldots+r_{m}} \cdot 2^{r_{1}+r_{3}+\ldots+r_{m} B}
\end{gathered}
$$

e quindi per la (23): $K<(8 a)^{m r_{1}} \cdot q^{\delta r_{1}}$, e infine per la (14):

$$
K\left(r_{1}+1\right)\left(r_{2}+1\right) \ldots\left(r_{m}+1\right) \leq K 2^{m r_{1}}<q_{1}{ }^{2 \delta r_{1}} .
$$

Giungiamo così, grazie alla (17), alla limitazione:

$$
F<q_{1}-\frac{m}{2}\left(\left(1-2(1+\sigma) \tau \mid\left\{1+\eta+f\left(q_{m}\right) \mid-\sigma \tau\right) r_{1}\right.\right.
$$

Confrontando la (26) e la (27) otteniamo:

$$
1+\eta+2(1+\sigma) \tau+\Omega>\{1-2(1+\sigma) \tau\}\left\{1+\eta+f\left(q_{m}\right)\right\}-\sigma \tau,
$$

e quindi :

$$
1+\eta+f\left(q_{m}\right)<\frac{1+\eta+(2+3 \sigma) \tau+\Omega}{1-2(1+\sigma) \tau}=\frac{1+\eta+2 \tau+3 \sigma \tau+\frac{\omega \tau}{2 g+1}}{1-2(1+\sigma) \tau}
$$

in evidente contrasto colla (20).

Da questa contraddizione risulta provato il teorema proposto. 